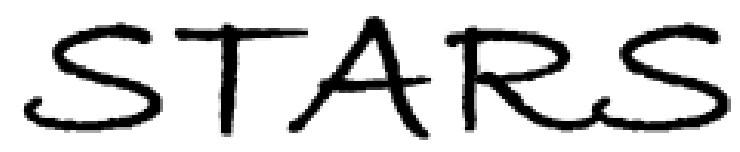

University of Central Florida

STARS

Faculty Bibliography 2000s

Faculty Bibliography

$1-1-2001$

\title{
Effect of spin-orbit interaction in photoionization of bromine
}

H. P. Saha

University of Central Florida

Dong Lin

University of Central Florida

Find similar works at: https://stars.library.ucf.edu/facultybib2000

University of Central Florida Libraries http://library.ucf.edu

This Article is brought to you for free and open access by the Faculty Bibliography at STARS. It has been accepted for inclusion in Faculty Bibliography 2000 s by an authorized administrator of STARS. For more information, please contactSTARS@ucf.edu.

\section{Recommended Citation}

Saha, H. P. and Lin, Dong, "Effect of spin-orbit interaction in photoionization of bromine" (2001). Faculty Bibliography 2000s. 8186.

https://stars.library.ucf.edu/facultybib2000/8186

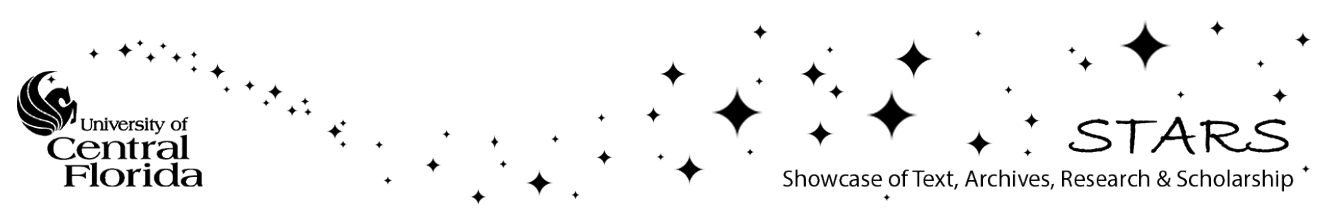




\title{
Effect of spin-orbit interaction in photoionization of bromine
}

\author{
H. P. Saha and Dong Lin \\ Department of Physics, University of Central Florida, Orlando, Florida 32816
}

(Received 11 September 2000; published 5 March 2001)

\begin{abstract}
The partial and total photoionization cross sections from the initial ${ }^{2} P_{3 / 2}$ ground state of atomic $\mathrm{Br}$ to the final $\mathrm{Br}^{+}{ }^{3} P_{J}$ components across the $4 p^{4}\left({ }^{1} D_{2}\right) 6 d, 8 s\left({ }^{2} P,{ }^{2} D,{ }^{2} F,{ }^{2} S\right)$ autoionization resonances are presented. The multiconfiguration Hartree-Fock (MCHF) method has been used to calculate both bound and continuum wave functions in the nonrelativistic limit. The Breit-Pauli approximation is applied to determine the effect of spin-orbit interactions in the photoionization of atomic $\mathrm{Br}$ with the MCHF wave function as input. Our calculated results are compared with experimental data.
\end{abstract}

DOI: 10.1103/PhysRevA.63.042701

PACS number(s): $32.80 . \mathrm{Fb}, 32.80 . \mathrm{Dz}$

\section{INTRODUCTION}

Recently there has been a growing interest in the photoionization of neutral halogens both experimentally and theoretically. Experiments of this type can provide partial cross sections for a transition, producing a particular fine-structure level of the residual ion. Such partial cross sections offer more severe tests of theoretical approximation methods than do the cross sections measured without regard to individual fine-structure levels. With the advent of several new synchrotron light sources around the world, which will permit experimentalists to study additional information on photoionization processes, a more detailed theoretical analysis with accurate methods is necessary.

Halogen systems are particularly important because of their use in chemical lasers as well as their great affinity for forming chemical compounds. Photoionization of chlorine has been investigated by many authors. However, photoexcitation and photoionization of bromine have received relatively poor attention. Theoretically, this is due to the fact that the target and the remaining core are an open shell atom and an ion, experimentally, it is due to its strong chemical reactivity. On the experimental side there have been studies by photoabsorption [1,2], photoemission [3,4], ion yield [5], He I photoelectron spectrometry [6-9], multiphoton ionization [10], and most recently electron emission [11-13]. Among these, the most recent and relevant works in the present investigation are the electron emission experiments by Benzaid and co-workers $[12,13]$. They present evidence of the $L S$-forbidden spin-orbit induced autoionization resonances $4 s^{2} 4 p^{4}\left({ }^{1} D_{2}\right) 6 d{ }^{2} F$ and ${ }^{2} S$, in addition to the allowed $4 s^{2} 4 p^{4}\left({ }^{1} D_{2}\right) 6 d{ }^{2} D,{ }^{2} P$ resonances, arising from $4 p$ $\rightarrow n s, m d$ excitations from the ground state $4 s^{2} 4 p^{5}{ }^{2} P_{3 / 2}$ of bromine, corresponding to a specific final spin-orbit state ${ }^{3} P_{2,1,0}$ of the ionic core. On the theoretical side, only Robicheaux and Greene [14] treated the autoionization regime using the eigenchannel $R$-matrix method [15]. They reported only the total cross section across the resonances, but obtained very good results.

In the present investigation we made a comprehensive study of the properties of the $4 s^{2} 4 p^{4}\left({ }^{1} D_{2}\right) 6 d$, $8 s$ resonances arising from $4 p$ photoexcitation of the bromine atom in the $4 s^{2} 4 p^{5}{ }^{2} P_{3 / 2}$ ground state. The analysis of these autoionization resonances allows us to understand the important as- pects of the electronic structure and dynamics of the electrons associated with the final ionic state $\mathrm{Br}^{+} 4 s^{2} 4 p^{4}\left({ }^{1} D_{2}\right)$ created in the decay of these resonances. The purpose of the present investigation is to understand and verify the experimental observation $[12,13]$ in detail as well as to test the validity of our newly developed multiconfiguration HartreeFock-Breit-Pauli (MCHF+BP) method [16] which includes relativistic effects into the bound and continuum wave functions in the form of the BP approximation.

Here we report a detailed theoretical analysis of the autoionization resonances, both $L S$ allowed and $L S$ forbidden, by calculating the partial as well as total photoionization cross sections from a $L_{0} S_{0} J_{0}$ initial bound state to an $L S J$ final continuum state corresponding to all exit channels. The photoinization matrix elements are then transformed to obtain partial photoionization cross sections corresponding to a specific fine-structure level of the ${ }^{3} P_{2,1,0}$ core ionic states for comparison with the experimentally determined partial cross sections. In the following we present the results of our investigation in the autoionization regime in the vicinity of $8 s$ and $6 d$ excitations from the first ionization threshold, $\mathrm{Br}^{+} 4 p^{4}\left({ }^{3} P_{2}\right)$, to the $\mathrm{Br}^{+} 4 p^{4}\left({ }^{1} D_{2}\right)$ threshold of the multiplet created by the ionization of a $4 p$ electron in $\mathrm{Br}$.

\section{THEORY}

\section{A. MCHF wave function for a continuum state}

The wave function for a scattering state with label $\gamma$, energy $E$, and term $L S$, in the multiconfiguration Hartree-Fock approximation, can be expressed as

$$
\begin{aligned}
\Psi(\gamma L S ; N+1)= & \sum_{j}^{m_{c}} a_{j} \Phi\left(\gamma_{j} L_{c} S_{c} ; N\right) \phi_{k l} \\
& +\sum_{i}^{m} c_{i} \Phi\left(\gamma_{i} L S ; N+1\right),
\end{aligned}
$$

where the first term represents a correlated $N$-electron target wave function coupled to a scattering electron. The $N$-electron target, that is an eigenstate of $L_{c}$ and $S_{c}$, is described in terms of $N$-electron bound configuration states $\Phi\left(\gamma_{j} L_{c} S_{c} ; N\right)$, with configuration $\gamma_{j}$ and term $L_{c} S_{c}$, mixing coefficient $a_{j}$, and total energy $E_{c}$ coupled to a scattering 
wave function $\phi_{k l}$ with orbital angular momentum $l$, to yield an antisymmetric configuration state for the $(N+1)$-electron system, with a final value $L S$ and a configuration $\gamma_{j} k l$. The second term $\Phi\left(\gamma_{i} L S ; N+1\right)$ is an $(N+1)$-electron bound configuration which is an eigenstate with the same $L$ and $S$, and which is included to allow for polarization and electroncorrelation effects. The above wave function is defined in terms of a set of radial bound and continuum wave functions $P_{i}(r), i=1,2, \ldots, m$.

The key to implementing the MCHF method is to select an appropriate collection of configurations (including both bound and continuum) for the wave function. The MCHF method optimizes both the bound and continuum wave functions simultaneously at each incident energy to account for electron correlation and polarization effects. In this approximation, all the radial functions are the solutions of the second order coupled integro-differential equations of the form

$$
\begin{aligned}
{\left[\frac{d^{2}}{d r^{2}}+\frac{2 Z}{r}-\frac{l_{i}\left(l_{i}+1\right)}{r^{2}}\right] P_{i}(r)=} & \frac{2}{r}\left[Y_{i}(r) P_{i}(r)+X_{i}(r)\right. \\
& \left.+I_{i}(r)\right]+\sum_{i^{\prime}} \varepsilon_{i i^{\prime}} P_{i^{\prime}}(r),
\end{aligned}
$$

where the off-diagonal energy parameters $\varepsilon_{i i^{\prime}}$ are related to Lagrange multipliers that ensure orthogonality assumptions.

In the MCHF method, the bound and the continuum radial functions are determined by solving the above set of coupled equations under proper boundary conditions. The bound radial functions satisfy the boundary conditions:

$$
P_{i}(r) \underset{r \rightarrow 0}{\sim} r^{l+1} \text { and } \quad P_{i}(r) \underset{r \rightarrow \infty}{\sim} 0 .
$$

In this case the diagonal energy parameter $\varepsilon_{i i}$, which is an eigenvalue of the integrodifferential equation, needs to be determined. The radial functions for the continuum orbital satisfy the conditions

$$
P_{i}(r) \underset{r \rightarrow 0}{\sim} r^{l+1}
$$

and

$$
P_{i}(r) \underset{r \rightarrow \infty}{\sim}\left(\frac{2}{\pi k}\right) \sin \left(k r-\frac{l \pi}{2}+\frac{q}{k} \ln (2 k r)+\sigma_{l}+\delta_{l}\right),
$$

where $k^{2}$ is the kinetic energy of the photoelectron, and $\varepsilon_{i i}$ $=-k^{2}$. The net charge of the ion is $q=\mathrm{Z}-\mathrm{N}, \sigma_{l}=\arg \Gamma(l$ $+1-i q / k)$ is the Coulomb phase shift, and $\delta_{l}$ is the residual phase shift.

The coefficients $c_{i}$ are solutions of the system of equations

$$
\begin{gathered}
\sum_{i^{\prime}}^{m}\left\langle\Phi_{i}|H-E| \Phi_{i^{\prime}}\right\rangle c_{i^{\prime}}+\sum_{j}^{m_{c}}\left\langle\Phi_{i}|H-E| \Phi_{j}\right\rangle a_{j}=0, \\
i=1,2, \ldots, m \\
\Phi_{j}=\Phi\left(\gamma_{j} L_{c} S_{c} ; N\right) \cdot \phi_{k l}, \quad j=1,2, \ldots, m_{c}
\end{gathered}
$$

$$
\begin{gathered}
\Phi_{i}=\Phi\left(\gamma_{i} L S ; N+1\right), \quad i=1,2, \ldots, m \\
E=E_{c}+\frac{k^{2}}{2} .
\end{gathered}
$$

The coupled integrodifferential equations are solved numerically by an iterative method.

\section{B. Breit-Pauli approximation}

The Breit-Pauli Hamiltonian for an $N$-electron system is given by

$$
H_{\mathrm{BP}}^{N}=H_{\mathrm{NR}}^{N}+H_{\mathrm{REL}}^{N}
$$

where $H_{\mathrm{NR}}^{N}$ is the usual nonrelativistic Hamiltonian,

$$
H_{\mathrm{NR}}^{N}=\sum_{i=1}^{N}\left(-\frac{1}{2} \nabla_{i}^{2}-\frac{Z}{r_{i}}\right)+\frac{1}{r_{i j}},
$$

and $H_{\mathrm{REL}}^{N}$ consists of one- and two-body relativistic correction terms resulting from the reduction of the Dirac equation and the Breit interaction to Pauli form. We can rewrite the equation as

$$
H_{\mathrm{BP}}^{N}=(\text { fine structure }+ \text { non-fine-structure }),
$$

where

$$
H_{\mathrm{SO}}^{N}+H_{\mathrm{SOO}}^{N}+H_{\mathrm{SS}}^{N}=(\text { fine structure })
$$

and

$$
\begin{aligned}
& \left(H_{\mathrm{NR}}^{N}+H_{\text {mass }}^{N}+H_{\mathrm{D} 1}^{N}+H_{\mathrm{OO}}^{N}+H_{\mathrm{D} 2}^{N}+H_{\mathrm{SSC}}^{N}\right) \\
& \quad=(\text { non-fine-structure })
\end{aligned}
$$

The non-fine-structure interactions commute with $\mathbf{L}^{2}, \mathbf{S}^{2}$, $\mathbf{L}_{z}$, and $\mathbf{S}_{z}$, while the fine-structure interactions only commute with $\mathbf{J}^{2}$ and $\mathbf{J}_{z}$.

\section{Photoionization cross sections}

Our calculation is based on the electric dipole approximation. The partial photoionization cross section as a function of photon energy $\omega$ for a transition from an initial bound state $i$ to a final state $f$ is given by

$$
\sigma(\omega)=4 \pi^{2} \alpha a_{0}^{2} \omega \sum_{f, m}\left|\left\langle\Psi_{f}|T| \Psi_{i}\right\rangle\right|^{2}
$$

where $\alpha$ is the fine-structure constant, and $a_{0}$ is the Bohr radius of the hydrogen atom. $\Psi_{i}$ and $\Psi_{f}$ are the initial- and the final-state wave functions, respectively, calculated in $L S$ coupling, and the sums run over final configurations and all magnetic quantum numbers.

The dipole transition operator $T$ is given by 


$$
T=T_{L}=\sum_{j=1}^{n} z_{j} \quad \text { in length form }
$$

and

$$
T=T_{V}=\sum_{j=1}^{n} \frac{\nabla_{z j}}{i \omega} \quad \text { in velocity form. }
$$

The cross-section results calculated in the length and velocity forms will be identical when $\Psi_{i}$ and $\Psi_{f}$ are exact solutions of the same Hamiltonian.

The partial photoionization cross section from the initial $L_{0} S_{0} J_{0}$ state to a specific $L_{c} S_{c} J_{c}$ final ionic state is given by [17]

$$
\begin{aligned}
\sigma_{J_{0} \rightarrow J_{c}}(\omega)= & \frac{4 \pi^{2} \omega}{c} \frac{\left[S_{0}\right]\left[J_{c}\right]}{\left[J_{0}\right]} \\
& \times \sum_{l j J L L^{\prime}}\left\langle L_{0} S_{0} J_{0}|T|\left(L_{c} l\right) L\left(S_{c} s\right) S J\right\rangle \\
& \times\left\langle L_{0} S_{0} J_{0}|T|\left(L_{c} l\right) L^{\prime}\left(S_{c} s\right) S J\right\rangle \\
& \times[L]^{1 / 2}\left[L^{\prime}\right]^{1 / 2}[J]\left\{\begin{array}{ccc}
L_{c} & l & L \\
S_{c} & s & S \\
J_{c} & j & J
\end{array}\right\} \\
& \times\left\{\begin{array}{ccc}
L_{c} & l & L^{\prime} \\
S_{c} & s & S \\
J_{c} & j & J
\end{array}\right\},
\end{aligned}
$$

where $L S J$ corresponds to final states. The sum over $j$ can be performed using a standard sum rule for the product of two $9 j$ symbols [18]. The final result is

$$
\begin{aligned}
& \sigma\left(J_{0}, J_{c}\right)=\frac{4 \pi^{2} \omega}{c\left[J_{0}\right]} \sum_{l, J, L, L^{\prime}}(-1)^{J+J_{c}-l-1 / 2}[L]^{1 / 2}\left[L^{\prime}\right]^{1 / 2}\left[S_{0}\right] \\
& \times\left[J_{0}\right]\left\langle\alpha_{0} L_{0} S_{0} J_{0}\left\|\sum_{j} r_{j}^{(1)}\right\| \alpha\left(L_{c} S_{c}\right) \varepsilon l L S J\right\rangle \\
& \times\left\langle\alpha_{0} L_{0} S_{0} J_{0}\left\|\sum_{j} r_{j}^{(1)}\right\| \alpha\left(L_{c} S_{c}\right) \varepsilon l L^{\prime} S J\right\rangle \\
& \times \sum_{x}[x]\left\{\begin{array}{ccc}
L & L^{\prime} & x \\
S_{0} & S_{0} & J
\end{array}\right\}\left\{\begin{array}{ccc}
S_{c} & S_{c} & x \\
S_{0} & S_{0} & \frac{1}{2}
\end{array}\right\} \\
& \times\left\{\begin{array}{ccc}
L & L^{\prime} & x \\
L_{c} & L_{c} & l
\end{array}\right\}\left\{\begin{array}{lll}
S_{c} & S_{c} & x \\
L_{c} & L_{c} & J_{c}
\end{array}\right\}
\end{aligned}
$$

where the curly brackets represent $6 j$ symbols.

\section{COMPUTATIONAL PROCEDURE}

The photoionization of a $4 p$ electron of atomic bromine creates a bromine ion in one of several multiplets. The photoionization occurs via autoionization resonances converging to different ionization thresholds. We study the photoionization processes

$$
\begin{aligned}
\hbar \omega & +\mathrm{Br} 4 s^{2} 4 p^{52} P_{3 / 2} \\
& \rightarrow \operatorname{Br} 4 s^{2} 4 p^{4}\left({ }^{1} D_{2}\right) 6 d, 8 s\left({ }^{2} P,{ }^{2} D,{ }^{2} F,{ }^{2} S\right) \\
& \rightarrow \operatorname{Br} 4 s^{2} 4 p^{4}\left({ }^{3} P_{2,1,0}\right)+k d, k s\left({ }^{2} P,{ }^{2} D,{ }^{2} F,{ }^{2} S\right),
\end{aligned}
$$

where the final states will couple in the LSJ scheme to ${ }^{2} P_{3 / 2,1 / 2},{ }^{2} D_{5 / 2,3 / 2},{ }^{2} F_{5 / 2}$, and ${ }^{2} S_{1 / 2}$. In this paper we are interested only in excitations from the ${ }^{2} P_{3 / 2}$ ground state and in the ${ }^{3} P_{2,1,0}$ core ionic states.

First of all, we use the MCHF method to calculate both bound [19] and continuum [20] wave functions for the initial and final states in $L S$ coupling. A variational calculation is performed for orbitals of the initial $4 s^{2} 4 p^{5}{ }^{2} P$ state of bromine using the MCHF method. The final ionic states $4 s^{2} 4 p^{4}\left({ }^{3} P,{ }^{1} D,{ }^{1} S\right)$ are obtained by a similar calculation, with the core orbitals the same as those for the ground state. For the continuum wave functions we perform calculations with correlated configurations for the resonance states using the multiconfiguration Hartree-Fock method for continuum wave functions and then perform a configuration-interaction calculation with the resonance states. In LSJ coupling, with the orbitals obtained in the $L S$ coupling, the Breit-Pauli interaction matrix is determined for a large set of configuration states, including a number of different $L S$ states for the initial bound and the final ionic core states. The eigenvalues and eigenvectors of this interaction matrix determine the total energy and wave function expansions, respectively, of the $L S J$ states. For the continuum calculation in $L S J$ coupling we modify [16] the Breit-Pauli program [21,22] for the bound state to perform calculations in the continuum states. In this case also, with the bound and continuum orbitals obtained in $L S$ coupling as input, a Breit-Pauli interaction matrix is formed with a large set of configuration states, including configurations from different $L S$ states. In this case the eigenvectors of the interaction matrix determine the wave function expansion of the $L S J$ states.

In the MCHF calculations for the wave functions in $L S$ coupling we included a large number of configurations so that the theoretical term energies would be in reasonable agreement with observation. For the initial state the variational MCHF calculation was over the set of 40 configurations states of the ${ }^{2} P$ term, namely,

$$
\left\{4 s^{2} 4 p^{5}, 4 s 4 p^{5} 4 d, 4 s^{2} 4 p^{4} 5 p, 4 s 4 p^{4} 4 d 5 p, 4 s 4 p^{4} 5 s 5 p, 4 p^{6} 5 p, 4 p^{5} 5 s^{2}, 4 p^{5} 5 p^{2}, 4 s^{2} 4 p^{3} 4 d^{2}, 4 s^{2} 4 p^{3} 5 s^{2}, 4 s^{2} 4 p^{3} 5 p^{2}\right\}^{2} P
$$


TABLE I. Comparison of energy levels (ionization thresholds) of $\mathrm{Br}^{+} 4 s^{2} 4 p^{4}$ with experiment.

\begin{tabular}{ccc}
\hline \hline State & Calculated $(\mathrm{eV})$ & Experiment $(\mathrm{eV})$ \\
\hline${ }^{3} P_{2}$ & 11.8382 & 11.8138 \\
${ }^{3} P_{1}$ & 12.1343 & 12.2027 \\
${ }^{3} P_{0}$ & 12.2805 & 12.2896 \\
${ }^{1} D_{2}$ & 13.1879 & 13.3127 \\
${ }^{1} S_{0}$ & 15.0177 & 15.2689 \\
\hline \hline
\end{tabular}

The inner $1 s, 2 s, 2 p, 3 s, 3 p, 3 d, 4 s$, and $4 p$ radial functions were kept fixed at their Hartree-Fock value. The MCHF calculation was performed for the ground state of the core ion $\mathrm{Br}^{+} 4 s^{2} 4 p^{4}{ }^{3} P$ with 12 configurations. The other ionic states were calculated similarly. In the LSJ calculation we added a few more configurations with the same $L S$ as well as different $L S$ in both the initial and core ion and each of the final continuum states. These configurations are selected according to their strength of mixing with the parent states. For each of the final continuum states, the LSJ expansion was over 200 configurations. The energies of each multiplet calculated in the $\mathrm{MCHF}+\mathrm{BP}$ approximation are tested and compared with experiment in Table I.

It should be noted that the present $\mathrm{MCHF}+\mathrm{BP}$ method is similar to the usual intermediate coupling approach. However, the advantage of this method lies in the fact that it allows one to calculate the partial photoionization cross sections from a specific fine-structure level of an initial state to a specific fine-structure level of an ionic state as well as to a specific fine-structure level of a final state.

\section{RESULTS}

Previously we reported [23] the partial photoionization cross sections calculated in $L S$ coupling, with relativistic effects, including the spin-orbit interaction, neglected. As a result, the $L S$-forbidden excited state resonances $\mathrm{Br}^{*}$ $4 s^{2} 4 p^{4}\left({ }^{1} D_{2}\right) 6 d^{2} F_{5 / 2}$ and ${ }^{2} S_{1 / 2}$ did not appear into the partial cross sections. In the present investigation we have included the relativistic effects, including the spin-orbit interaction, through the Breit-Pauli approximation. In Figs. 1, 2, and 3 we display partial photoionization cross sections for specific $L S J$ final states, and their sum, corresponding to two different exit channels $4 p^{4}\left({ }^{3} P\right) k d$ and $4 p^{4}\left({ }^{3} P\right) k s$. These will provide information about which specific final $L S J$ state corresponding to specific exit channel is responsible for different autoionization resonances. The relative heights of these resonances will determine the strength of the interaction of the bound excited channels with the specific final LSJ state corresponding to a specific autoionization resonance.

The partial photoionization cross sections corresponding to the exit channel $4 s^{2} 4 p^{4}\left({ }^{3} P\right) k d$ and the final ${ }^{2} D_{5 / 2}$ and ${ }^{2} D_{3 / 2}$ states and their sum are depicted separately in Fig. 1. The spectra represent two autoionization resonances in each of these final $L S J$ states. In the ${ }^{2} D_{5 / 2}$ final state they include the $\mathrm{Br}^{*} 4 s^{2} 4 p^{4}\left({ }^{1} D_{2}\right) 6 d^{2} F_{5 / 2}$ and $\mathrm{Br}^{*}$ $4 s^{2} 4 p^{4}\left({ }^{1} D_{2}\right) 8 s^{2} D_{5 / 2}$ excited states. These resonances appear due to the interaction of the $4 s^{2} 4 p^{4}\left({ }^{3} P\right) k d{ }^{2} D_{5 / 2}$ final
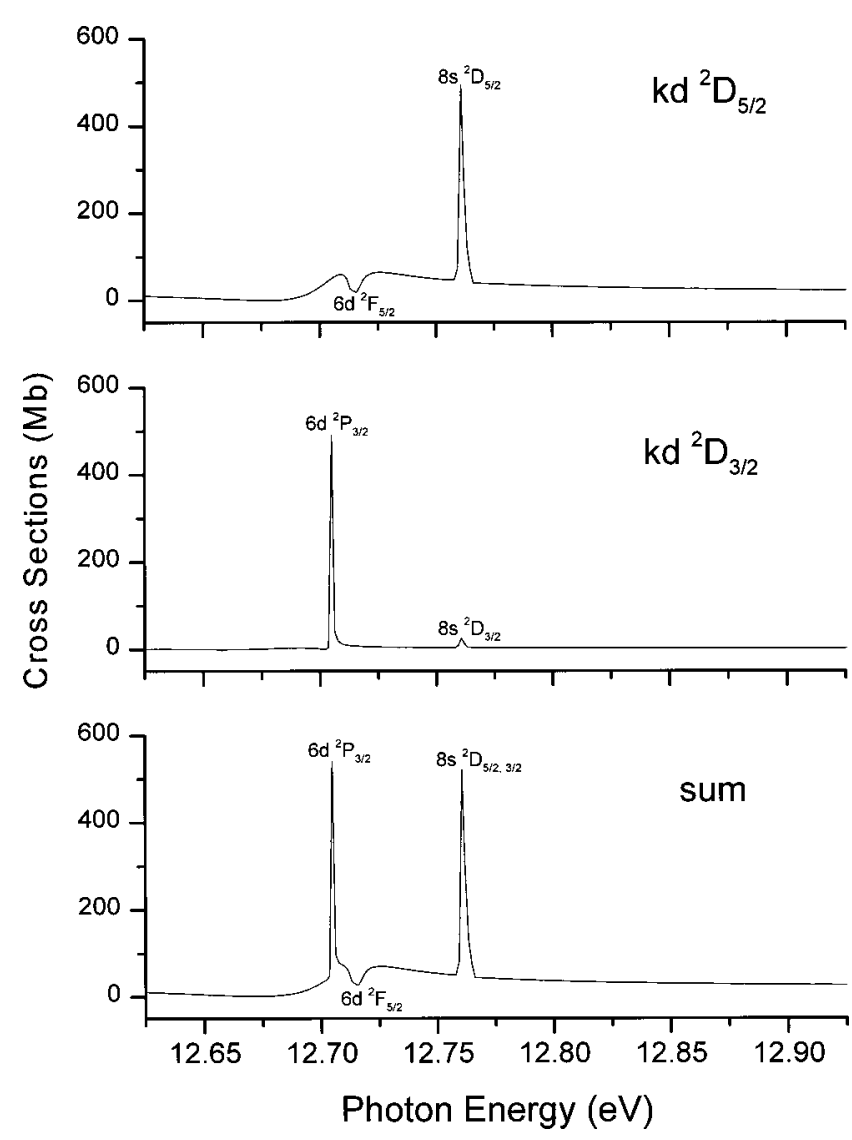

FIG. 1. Partial photoionization cross sections of bromine corresponding to the $\mathrm{Br} 4 s^{2} 4 p^{4}\left({ }^{3} P\right) k d^{2} D_{5 / 2},{ }^{2} D_{3 / 2}$ final states, and their sum.

state with the $\mathrm{Br}^{*} 4 s^{2} 4 p^{4}\left({ }^{1} D_{2}\right) 6 d{ }^{2} F_{5 / 2}$ and $\mathrm{Br}^{*}$ $4 s^{2} 4 p^{4}\left({ }^{1} D_{2}\right) 8 s^{2} D_{5 / 2}$ excited bound channels. The ${ }^{2} F_{5 / 2}$ resonance is $L S$ forbidden. The excitation of ${ }^{2} F_{5 / 2}$ can proceed only by spin-orbit coupling, but its decay into all three $4 s^{2} 4 p^{4}\left({ }^{3} P_{2,1,0}\right)$ ionic core states is $L S$ allowed, whereas both excitation and decay of ${ }^{2} D_{5 / 2}$ resonance state are $L S$ allowed. The ${ }^{2} F_{5 / 2}$ resonance is windowlike, which verifies the experimental observation $[12,13]$ and corresponds to having a $q$ parameter close to zero in the Fano formalism [24]. However, in the ${ }^{2} D_{3 / 2}$ final state both $\mathrm{Br}^{*} 4 s^{2} 4 p^{4}\left({ }^{1} D_{2}\right) 6 d{ }^{2} P_{3 / 2}$ and $8 s^{2} D_{3 / 2}$ autoionization resonances are $L S$ allowed, and are generated due to interaction of the $k d{ }^{2} D_{3 / 2}$ final state with the $6 d^{2} D_{3 / 2}$ and $8 s^{2} P_{3 / 2}$ excited bound channels.

In Fig. 2 the partial photoionization cross sections for the exit channel $4 s^{2} 4 p^{4}\left({ }^{3} P\right) k d$ and the final states ${ }^{2} P_{3 / 2}$ and ${ }^{2} P_{1 / 2}$ and their sum are shown. Two autoionization features, $\mathrm{Br}^{*} 4 s^{2} 4 p^{4}\left({ }^{1} D_{2}\right) 6 d{ }^{2} D_{3 / 2}$ and $8 s^{2} D_{3 / 2}$, appear in the $4 s^{2} 4 p^{4}\left({ }^{3} P\right) k d{ }^{2} P_{3 / 2}$ final state. The interaction of the $4 s^{2} 4 p^{4}\left({ }^{3} P\right) k d{ }^{2} P_{3 / 2}$ final state with the $8 s^{2} D_{3 / 2}$ excited bound channel is very small compared to that with $6 d^{2} D_{3 / 2}$ excited bound channel. In this case the excitation and decay processes for both resonances are $L S$ allowed. On the other hand, there is only one feature $\mathrm{Br}^{*} 4 s^{2} 4 p^{4}\left({ }^{1} D_{2}\right) 6 d{ }^{2} S_{1 / 2}$ appearing in the $\mathrm{Br} 4 s^{2} 4 p^{4}\left({ }^{3} P\right) k d{ }^{2} P_{1 / 2}$ final state. The $\mathrm{Br}^{*}$ $4 s^{2} 4 p^{4}\left({ }^{1} D_{2}\right) 6 d{ }^{2} S_{1 / 2}$ autoionization resonance is again $L S$ forbidden. Although the excitation of this resonance from the 


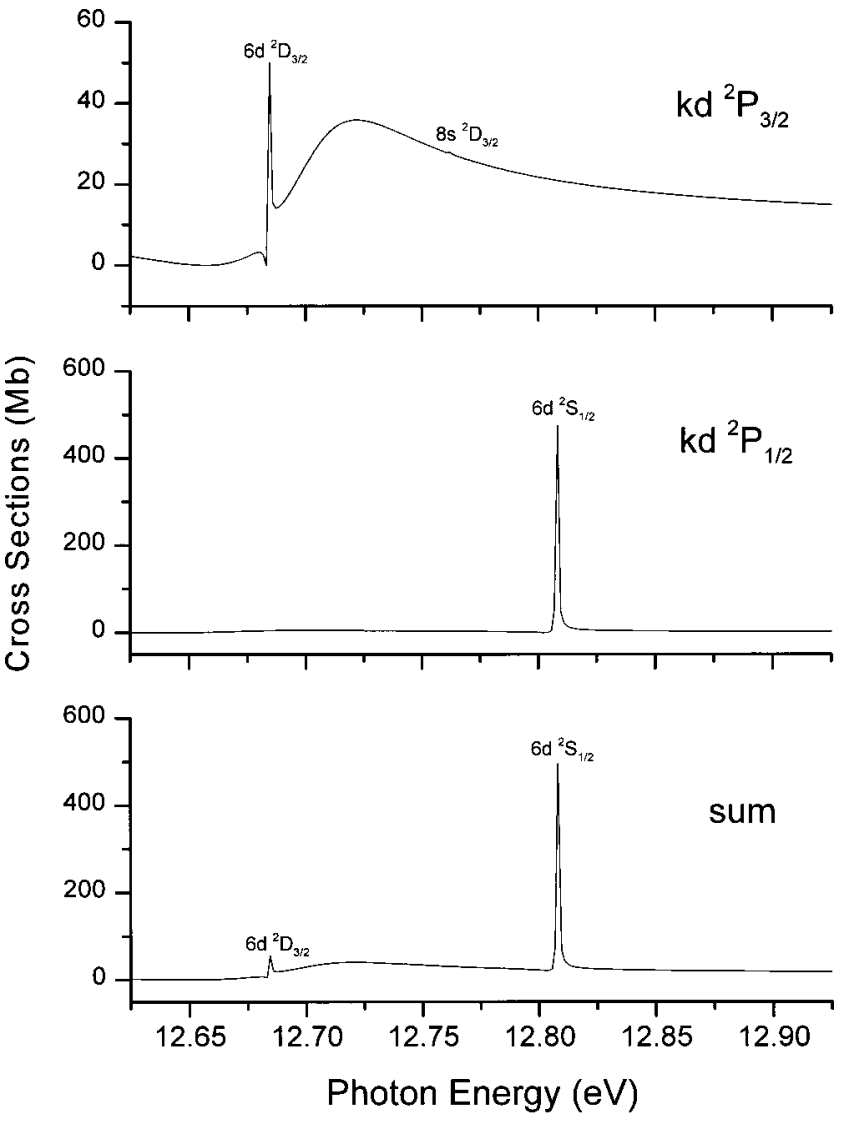

FIG. 2. Same as Fig. 1, but corresponding to the $\mathrm{Br}$ $4 s^{2} 4 p^{4}\left({ }^{3} P\right) k d{ }^{2} P_{1 / 2},{ }^{2} P_{3 / 2}$ final states, and their sum.

$4 s^{2} 4 p^{5}{ }^{2} P_{3 / 2}$ ground state is $L S$ allowed, spin-orbit interaction is required if the state is to decay into the $4 s^{2} 4 p^{4}\left({ }^{3} P_{2,1,0}\right)$ core ionic states via the coupling of the $4 s^{2} 4 p^{4}\left({ }^{1} D_{2}\right)$ and the ${ }^{3} P_{2,1,0}$ core ionic states.

Figure 3 presents the partial photoionization cross sections for the $4 s^{2} 4 p^{4}\left({ }^{3} P\right) k s$ exit channel corresponding to final ${ }^{2} P_{3 / 2}$ and ${ }^{2} P_{1 / 2}$ states. In the cross sections for the ${ }^{2} P_{3 / 2}$ final-state one-autoionization resonance, $\mathrm{Br}^{*}$ $4 s^{2} 4 p^{4}\left({ }^{1} D_{2}\right) 6 d{ }^{2} P_{3 / 2}$ which is $L S$ allowed, is clearly seen, whereas in the cross section for the ${ }^{2} P_{1 / 2}$ final state two autoionization resonances, $4 s^{2} 4 p^{4}\left({ }^{1} D_{2}\right) 6 d^{2} P_{1 / 2}$ and $4 s^{2} 4 p^{4}\left({ }^{1} D_{2}\right) 6 d^{2} S_{1 / 2}$, are clearly separated. As indicated earlier, the appearance of the $4 s^{2} 4 p^{4}\left({ }^{1} D_{2}\right) 6 d{ }^{2} S_{1 / 2}$ state is $L S$ forbidden, but both excitation and decay of $6 d{ }^{2} P_{1 / 2}$ resonance are $L S$ allowed. The energies for all of these autoionization resonances are presented in Table II.

In Fig. 4 the partial photoionization cross sections of bromine from the initial ${ }^{2} P_{3 / 2}$ state are shown for three specific ionic channels, $\mathrm{Br}^{+}\left({ }^{3} P_{2}\right), \mathrm{Br}^{+}\left({ }^{3} P_{1}\right)$, and $\mathrm{Br}^{+}\left({ }^{3} P_{0}\right)$. These cross sections are obtained from the $L_{0} S_{0} J_{0} \rightarrow L S J$ dipole matrix elements between the initial $L_{0} S_{0} J_{0}$ state to specific LSJ final states by the transformations described in the theory section. The advantage of our $\mathrm{MCHF}+\mathrm{BP}$ method is that we can compute data from a specific initial $L_{0} S_{0} J_{0}$ state to a specific $L S J$ final state. Then by a transformation [17] the partial cross section from a specific $J_{c}$ level of the core ion can be determined. In the energy region considered in the
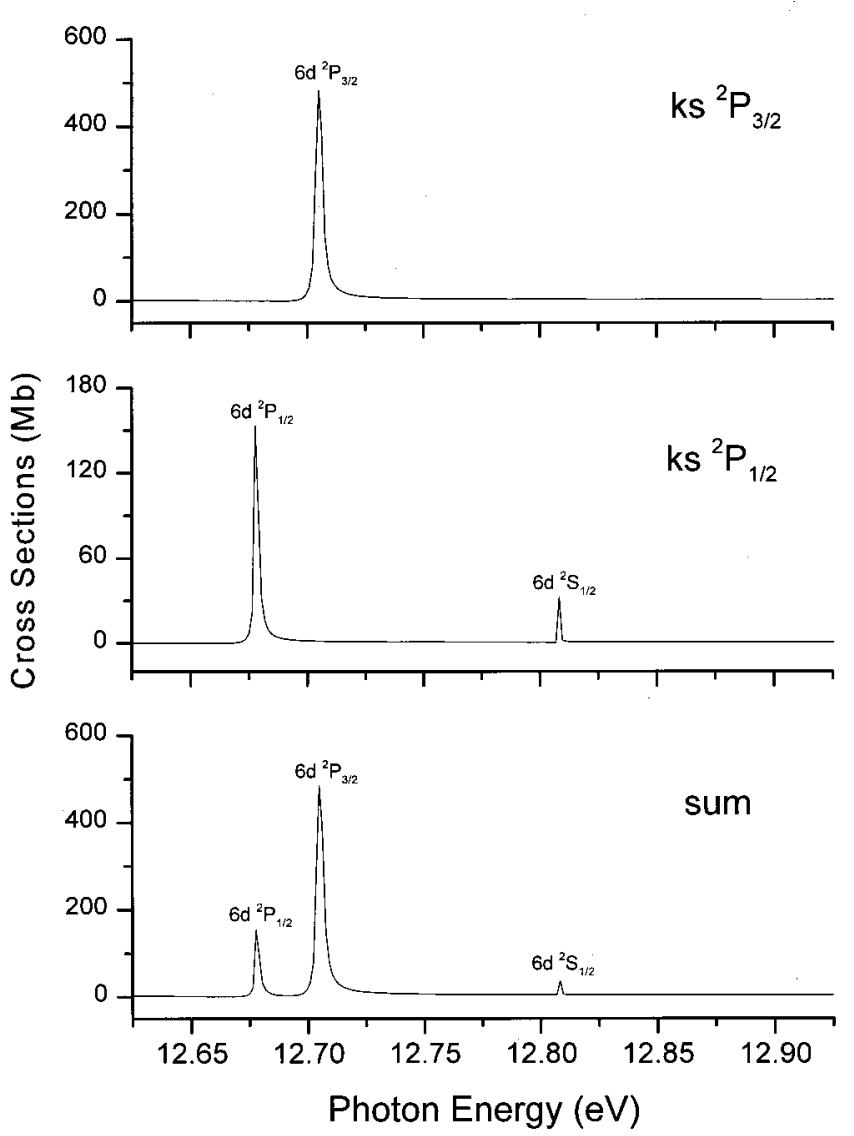

FIG. 3. Same as Fig. 1, but corresponding to the $\mathrm{Br}$ $4 s^{2} 4 p^{4}\left({ }^{3} P\right) k s{ }^{2} P_{1 / 2},{ }^{2} P_{3 / 2}$ final states, and their sum.

calculation the autoionization resonances appeared in each of the $4 s^{2} 4 p^{4}\left({ }^{3} P_{2,1,0}\right)$ ionic channels. They include the $\mathrm{Br}^{*} 4 s^{2} 4 p^{4}\left({ }^{1} D_{2}\right) 6 d\left({ }^{2} P_{3 / 2},{ }^{2} D_{3 / 2},{ }^{2} F_{5 / 2},{ }^{2} S_{1 / 2}\right)$ and $\mathrm{Br}^{*}$ $4 s^{2} 4 p^{4}\left({ }^{1} D_{2}\right) 8 s^{2} D_{3 / 2,5 / 2}$ resonances in the fine-structure level $J_{c}=2$ of the final ${ }^{3} P$ core ionic state. $8 s^{2} D_{3 / 2}$ and ${ }^{2} D_{5 / 2}$ resonances overlap. Out of these five autoionization resonances, three resonances $6 d{ }^{2} P_{3 / 2},{ }^{2} D_{3 / 2}$, and $8 s$ ${ }^{2} D_{3 / 2,5 / 2}$ are $L S$ allowed, and two resonances $6 d{ }^{2} F_{5 / 2}$ and $6 d{ }^{2} S_{1 / 2}$ are $L S$ forbidden, as indicated earlier. There are

TABLE II. Comparison of the resonance energies $(\mathrm{eV})$ for $\mathrm{Br}^{*} 4 s^{2} 4 p^{4}\left({ }^{1} D_{2}\right) 6 d, 8 s$ with experiment. ${ }^{*} J$ averaged.

\begin{tabular}{rcc}
\hline \hline State & Calculated & Experiment \\
\hline $6 d^{2} P_{1 / 2}$ & 12.6777 & \\
& $(12.6958)^{*}$ & 12.693 \\
${ }^{2} P_{3 / 2}$ & 12.7049 & \\
${ }^{2} D_{3 / 2}$ & 12.6845 & \\
& $(12.7033)^{*}$ & 12.693 \\
${ }^{2} D_{5 / 2}$ & 12.7133 & \\
${ }^{2} F_{5 / 2}$ & 12.7158 & 12.709 \\
${ }^{2} S_{1 / 2}$ & 12.8083 & 12.796 \\
$8 s{ }^{2} D_{3 / 2}$ & 12.7607 & 12.752 \\
${ }^{2} D_{5 / 2}$ & $(12.7607)^{*}$ & \\
\hline \hline
\end{tabular}



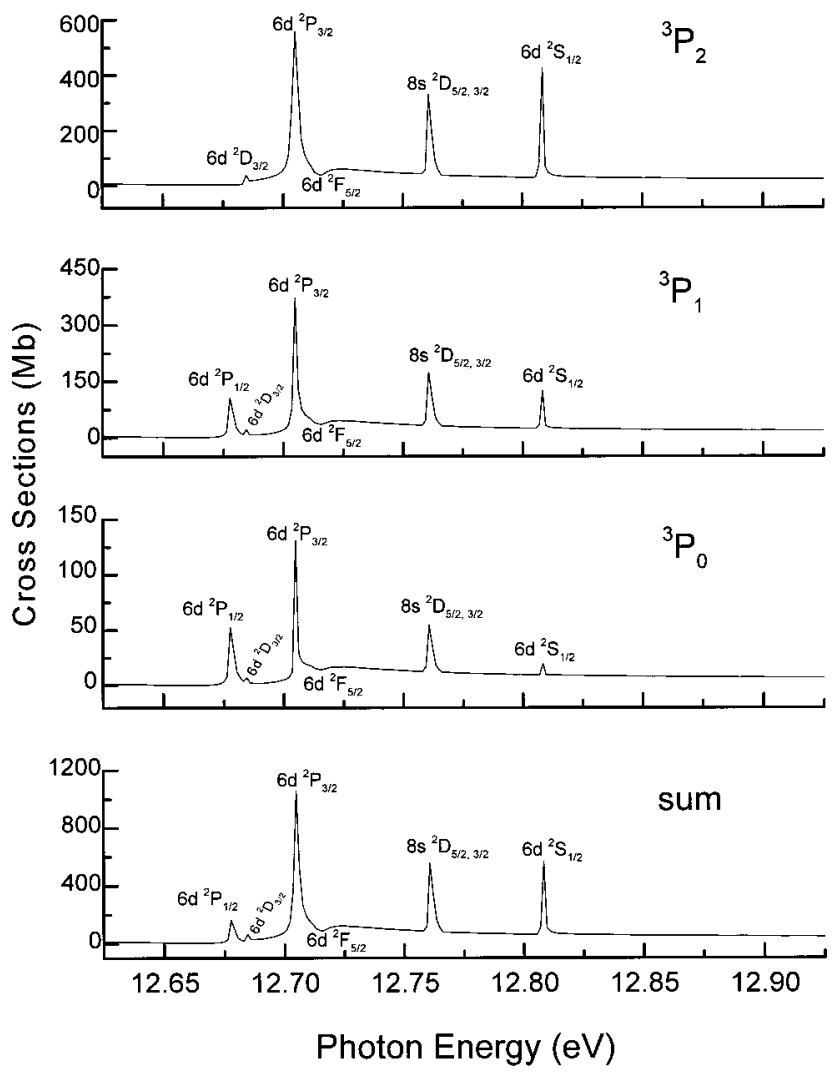

FIG. 4. Partial photoionization cross sections of bromine with respect to the $\mathrm{Br}^{+} 4 s^{2} 4 p^{4}{ }^{3} P_{2},{ }^{3} P_{1},{ }^{3} P_{0}$ core ionic states and their sum.

six autoionization resonances, $\mathrm{Br}^{*} 4 s^{2} 4 p^{4}\left({ }^{1} D_{2}\right) 6 d$ $\left({ }^{2} P_{1 / 2},{ }^{2} P_{3 / 2},{ }^{2} D_{3 / 2},{ }^{2} F_{5 / 2},{ }^{2} S_{1 / 2}\right)$ and $\mathrm{Br}^{*} 4 s^{2} 4 p^{4}\left({ }^{1} D_{2}\right) 8 s$ ${ }^{2} D_{3 / 2,5 / 2}$, corresponding to $J_{c}=1$ and 0 of the final ${ }^{3} P$ ionic state. Again four resonances $6 d{ }^{2} P_{1 / 2},{ }^{2} P_{3 / 2},{ }^{2} D_{3 / 2}$ and $8 s$ ${ }^{2} D_{3 / 2,5 / 2}$ are $L S$ allowed, and $6 d^{2} F_{5 / 2}$ and $6 d{ }^{2} S_{1 / 2}$ are $L S$ forbidden. The cross sections and heights of each of the autoionization resonances gradually decrease with the decrease in the fine-structure level $J_{c}$ of the final ionic state.

In the the bottom panel of Fig. 4 the total photoionization cross section of bromine leading to the $\mathrm{Br}^{+} 4 s^{2} 4 p^{4}\left({ }^{3} P_{2,1,0}\right)$ core ionic states are shown. The cross section clearly shows six autoionization resonances. All resonance spectra clearly exhibit the $L S$-forbidden $6 d^{2} F_{5 / 2}$ and ${ }^{2} S_{1 / 2}$ resonances at 12.7158 and $12.8083 \mathrm{eV}$, respectively, in addition to the $L S$ allowed $6 d{ }^{2} P_{1 / 2},{ }^{2} P_{3 / 2},{ }^{2} D_{3 / 2}$, and $8 s{ }^{2} D_{5 / 2},{ }^{2} D_{3 / 2}$ resonances. As indicated earlier, the ${ }^{2} F_{5 / 2}$ and ${ }^{2} S_{1 / 2}$ autoionization resonances are $L S$ forbidden. However, the two decay processes associated with ${ }^{2} F_{5 / 2}$ and ${ }^{2} S_{1 / 2}$ resonances are different. In the case of the ${ }^{2} F$ autoionization resonance, it is the excitation of ${ }^{2} F$ that depends on spin-orbit coupling, with the decay being allowed in $L S$ coupling, whereas the excitation of ${ }^{2} S$ is $L S$ allowed, with the decay being spin orbit induced.

In the $L S$ coupling calculation performed earlier, where relativistic effects including the spin-orbit interaction were neglected, only two $L S$-allowed resonances $6 d^{2} D$ and $8 s$ ${ }^{2} D$ were found corresponding to each of the ${ }^{3} P_{2,1,0}$ ionic states. As regards the background cross sections, in the two

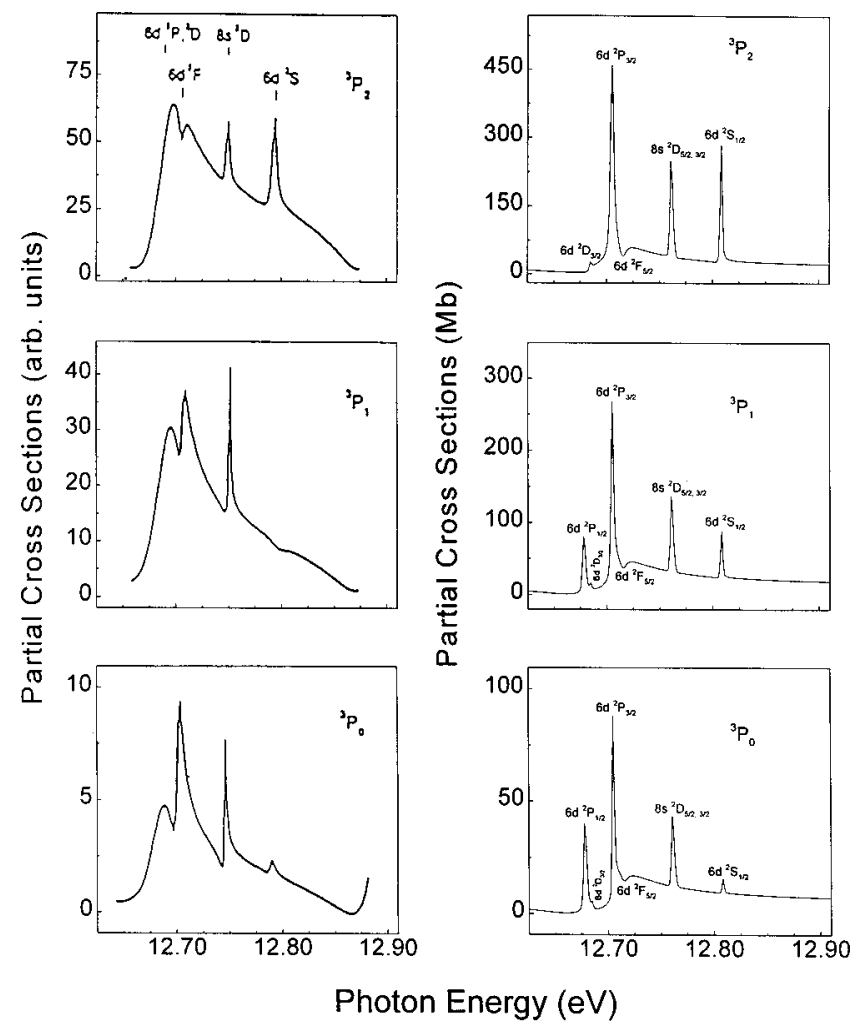

FIG. 5. Comparison of partial photoionization cross sections (right figure) for the $\mathrm{Br}^{+}\left({ }^{3} P_{2,1,0}\right)$ components with experiment (Refs. [12] and [13]) (left figure) across the $4 s^{2} 4 p^{4}\left({ }^{1} D_{2}\right) 6 d, 8 s$ resonances.

calculations, it is found that the cross sections are larger in the relativistic calculation compared to that in the $L S$ coupling. This may be attributed to the inclusion of larger set of configurations and the Breit-Pauli contributions in the relativistic calculation.

In Fig. 5 autoionization spectra obtained by experiment [13] are shown for all $J$ components of the $4 s^{2} 4 p^{4}\left({ }^{3} P_{2,1,0}\right)$ core ion along with the calculated data. Comparison of the experimental data in Fig. 5(a) with the calculated results in Fig. 5(b) shows that the positions of the resonances for all the $J$ components are in excellent agreement with the experiment. However, the experimental spectra are shown for $J$-averaged final excited resonance states whereas the calculated spectra shown correspond to each of the LSJ final excited resonance states. In the present calculation the decay strength of the $6 d{ }^{2} S$ resonance decreases from the $J_{c}=2$ to $J_{c}=0$ ionic channels. In the experiment $[12,13]$ it is seen that the entire decay strength is seen to be in the ${ }^{3} P_{2}$ ionic channel. A weak interference structure is observed in the ${ }^{3} P_{0}$ channel and a barely identifiable feature in the ${ }^{3} P_{1}$ channel. In Fig. 6, the total photoionization cross sections are compared with experiment. The energy positions of the resonance spectra agree very well with experiment. The rapid decrease of the background cross sections in the experimental results in both Figs. 5 and 6 may be attributed to the interaction of continuum-continuum channels in the calculation of the final state wave function. The MCHF method did not take into account the effect of the continuum-continuum interaction in the present calculation. 


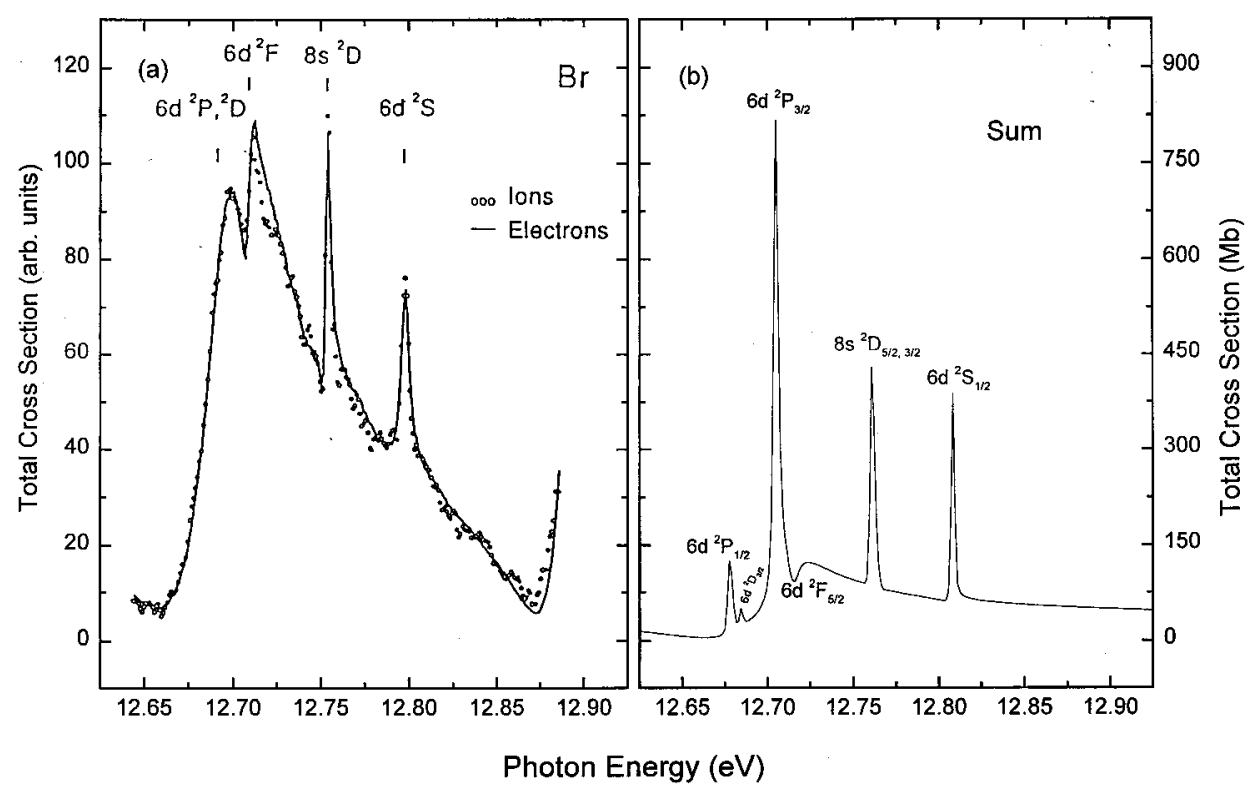

FIG. 6. Comparison of the sum of the cross section for the three components shown in Fig. 5(a) Experimental (Refs. [12] and [13]) cross section. (b) MCHF length cross section.

\section{CONCLUSION}

We obtained both $L S$-allowed and $L S$-forbidden autoionization resonances converging to the ${ }^{1} D_{2}$ threshold using the $\mathrm{MCHF}+\mathrm{BP}$ approximations. In this paper, we presented detailed information showing evidence of $L S$-forbidden autoionization resonances in the $L S J$ specific partial photoionization cross sections as well as the relative strengths of the population of each resonance state specific to each $J$ level of the final core ionic states. The experimental evidence for the spin-orbit induced $4 p^{4}\left({ }^{1} D_{2}\right) 6 d{ }^{2} F$ and ${ }^{2} S$ resonances converging to the ${ }^{1} D_{2}$ ionization threshold was analyzed and verified by a detailed calculation. The LSJ calculation allowed us to determine the differences in the dynamics between the $L S$-forbidden ${ }^{2} F$ and ${ }^{2} S$ autoionization resonances, which are consequences of spin-orbit interaction in the ${ }^{3} P_{2,1,0}$ partial cross sections. We determined energy po- sitions for each of the autoionization resonances, which are in excellent agreement with experiment.

Finally, we conclude that this study not only tests the reliability of our $\mathrm{MCHF}+\mathrm{BP}$ method, but also offers a comprehensive study of the spectroscopic and dynamic properties of the autoionization resonances involving the excitations $4 p \rightarrow 8 s, 6 d$, and ionization into the final core ionic states. The present investigation may provide a useful basis for this kind of phenomena in other open-shell atoms, and encourage experimental determinations to be undertaken explicitly at the fine-structure level.

\section{ACKNOWLEDGMENTS}

We thank Dr. F. Robicheaux for valuable discussions. This research was supported in part by the National Science Foundation.
[1] R. E. Huffman, J. C. Larrabee, and Y. Tanaka, J. Chem. Phys. 47, 856 (1967).

[2] V. N. Sarma and Y. N. Joshi, J. Phys. B 16, 1671 (1983).

[3] J. L. Tech, J. Res. Natl. Bur. Stand., Sect. A 67, 505 (1963).

[4] W. C. Martin and J. L. Tech, J. Opt. Soc. Am. B 51, 591 (1963).

[5] B. Ruscic, J. P. Greene, and J. Berkowitz, J. Phys. B 17, 1503 (1984).

[6] D. M. De Leeuw, R. Mooyman, and C. A. de Lange, Chem. Phys. Lett. 54, 231 (1978).

[7] K. Kimura, T. Yamasaki, and Y. Achiba, Chem. Phys. Lett. 58, 104 (1978).

[8] W. J. van der Mier, P. van der Meulen, and C. A. de Lange, Chem. Phys. 115, 109 (1987).
[9] J. B. Peel and E. I. von Nagy-Felsobuki, Spectrochim. Acta, Part B 43, 217 (1988).

[10] B. G. Koenders, K. E. Drabe, and C. A. de Lange, Chem. Phys. Lett. 138, 1 (1987).

[11] P. van der Meulen, M. O. Krause and C. A. Lange, J. Phys. B 25, 97 (1992).

[12] S. Benzaid, A. Menzel, J. Jimenez-Mier, S. J. Schaphorst, M. O. Krause, and C. D. Caldwell, Phys. Rev. A 54, R2537 (1996).

[13] S. Benzaid, M. O. Krause, A. Menzel, and C. D. Caldwell, Phys. Rev. A 57, 4420 (1998).

[14] F. Robicheaux and C. H. Greene, Phys. Rev. A 46, 3821 (1992).

[15] C. H. Greene, Phys. Rev. A 32, 1880 (1985). 
[16] H. P. Saha (unpublished).

[17] Cheng Pan and A. F. Starace, Phys. Rev. A 47, 295 (1993), and references therein.

[18] A. P. Yutsis, I. B. Levinson, and V. V. Vanagas, The Theory of Angular Momentum (Israel Program for Scientific Translation, Jerusalem, 1962).
[19] C. Froese Fischer, Comput. Phys. Commun. 14, 145 (1978).

[20] H. P. Saha (unpublished).

[21] C. Froese Fischer and H. P. Saha, J. Phys. B 17, 943 (1984).

[22] C. Froese Fischer and H. P. Saha, Phys. Scr. 32, 181 (1985).

[23] Dong Lin and H. P. Saha, Phys. Rev. A 59, 3614 (1999).

[24] U. Fano, Phys. Rev. 124, 1866 (1961). 